\title{
Effectiveness of Pharmacist-led Anticoagulation Management on Clinical Outcomes: A Systematic Review and Meta-Analysis.
}

\author{
Kelu Hou, Hui Yang, Zhikang Ye, Ying Wang, Lihong Liu, Xiangli Cui \\ Department of Pharmacy, Beijing Chao-Yang Hospital, Capital Medical University, Beijing, China
}

Received October 22, 2017; Accepted, November 6, 2017, Published, November 7, 2017.

\begin{abstract}
OBJECTIVES: We performed this systematic review and meta-analysis to confirm whether patients benefit more from pharmacist-led anticoagulation management than other models. METHODS: We searched PubMed, Embase, Cochrane Library and reference lists of yielded results conducted up to April 25, 2017. RCTs and observational cohort studies and case-control studies which compared the percentage of time within the target therapeutic range (TTR), the percentage of time within the expanded therapeutic range (TER), haemorrhage events, thrombosis events, mortality, patient satisfaction and/or medicine cost saving of pharmacist-led anticoagulation management with other models, and species were limited to humans. Two investigators evaluated methodology and extracted data from included studies independently. Data analysis were performed by STATA 12.0 software and quality of evidence assessment was performed by GRADEprofiler software. RESULTS: 8 RCTs and 9 observational cohort studies with 9919 patients were included eventually with high quality and no publication bias. In RCTs pooled results, TTR $(p=0.548$ moderate-quality), TER ( $p=0.285$, moderate-quality), total haemorrhage events ( $p=0.140$, low-quality), minor haemorrhage events ( $p=0.162$, low-quality), major haemorrhage events $(p=0.237$, low-quality), thrombosis events ( $p=0.615$, low-quality) and mortality ( $p=0.876$, low-quality) was not significant between two groups. In observational studies pooled results, TTR ( $p=0.000$, low-quality) was significant higher in pharmacist-led management group and the risk of total haemorrhage events ( $p=0.000$, moderate-quality), minor haemorrhage events ( $p=0.000$, moderate-quality) and thrombosis events $(p=0.000$, moderate-quality) were significant lower in pharmacist-led management group. Patient satisfaction and medicine cost saving were descriptively reviewed. CONCLUSIONS: According to the grading of evidence, we concluded that the risk of total haemorrhage events, minor haemorrhage events and thrombosis events significantly decreased in pharmacistled anticoagulation management group compared with other management models and no significant difference in TTR, TER, major haemorrhage events and mortality between two groups. Longer follow-up period RCT studies with large sample size should be done in the future to confirm effectiveness of pharmacist-led anticoagulation management model.
\end{abstract}

This article is open to POST-PUBLICATION REVIEW. Registered readers (see "For Readers") may comment by clicking on ABSTRACT on the issue's contents page.

\section{INTRODUCTION}

Warfarin is effectively used worldwide in prevention and treatment of thromboembolic diseases, which is commonly prescribed for the primary and secondary prevention and treatment of prosthetic heart valves, atrial fibrillation, pulmonary embolism, deep vein thrombosis, stroke, cardiomyopathy and myocardial infarction With approximately 2 million people started warfarin therapy in America every year (1), narrow therapeutic window, high individual dose variability and adverse events are challenging clinical practice and patient management in warfarin therapy. Genetic variants can be explained part of dose variability, such as variants of the CYP2C9, VKORC1, CYP4F2 genes. Some epigenetic factors, additional drug-drug interactions, patient behaviors, including diet, exercise and compliance are also potential sources (2). Patients on long-term warfarin therapy have a risk of $1 \% \sim 3 \%$ per year for haemorrhage leading to hospitalization or death $(3,4)$, which is the most worrying adverse events of warfarin. In order to achieve anticoagulation goal and avoid adverse events, the

Corresponding Authors: Xiangli Cui, Department of Pharmacy, Beijing Chao-Yang Hospital, 8 Gongren Tiyuchang Nanlu, Chaoyang District, Beijing, China, Email:ningning@ccmu.edu.cn; Lihong, Liu, Department of Pharmacy, Beijing Chao-Yang Hospital, 8 Gongren Tiyuchang Nanlu, Chaoyang District, Beijing, China, Email: liulihong@bjcyh.com. 
International Normalized Ratio (INR) is monitoring in a therapeutic range, usually kept in the range of 2.0-3.5 according to different indications (5). Anticoagulation management service (AMS) has been established several decades to ease heavy burden for primary physicians in oral anticoagulation therapy. It has been reported that patients managed by AMS or anticoagulation clinic (AC) had better treatment results, especially lower major bleeding rates than personal physician management (6-9). In addition, several studies reported significant improvements in haemorrhage and thrombosis events with patients in AMS (10-12). AMS could be managed by pharmacists, physicians, nurses and patient self-management in different models to finish routine work (13). Pharmacists were trained for dosing adjustment, drug-drug or drug-food interaction identification and adverse drug events monitoring on the foundation of pharmacy knowledge. In that pharmacists arecompetent to finish routine anticoagulation management issues and answer patient consults independently. There are more and more studies comparing pharmacist-led anticoagulation therapy with other management models and several studies reported higher INR control rate and less bleeding and thrombosis events in pharmacistmanaged patients. Nevertheless, some studies showed no significant difference between pharmacist management and other management models in INR control results $(14,15)$. Saokaew et al performed a meta-analysis that including RCTs, cohort studies and quasi-experimental studies in 2010 which found pharmacist-participated warfarin therapy management (PWTM) had statistically significant effects on the prevention of total bleeding of RCTs and non-RCTs (16). Another meta-analysis that including eight randomized controlled trials (RCTs) by Zhou et al showed a significant difference between pharmacist-managed care and other management models for satisfaction and the percentage of time within the standard therapeutic range, but no significant improvement on the bleeding and thrombosis events (17). However, some issues still need to be resolved. In that, we extracted data more strictly and completely and focused on the definition of each outcomes in different included studies in this meta-analysis. Moreover, high quality observational cohort and case-control studies that assessed validly were included to detect effects of pharmacist-led anticoagulation management. Above all, it's necessary to perform this systematic review and meta-analysis to evaluate the effectiveness pharmacist-led anticoagulation management including both RCTs and observational studies.

\section{METHODS}

Study Selection

Potential eligible studies were searched in PubMed, Embase, Cochrane Library and ClinicalTrials.gov for study reported effectiveness and safety of pharmacist-managed oral anticoagulation therapy. The search was performed from inception to April 25 2017.The key search terms were following: "pharmacist", "anticoagulant", "warfarin", "international normalized ratio". There was no limitation in the language of publications and study species were limited to humans. Two independent authors K.L.H. and X.L.C. performed study selection respectively, which included articles searching, deleting duplicate results, screening titles and abstracts to delete relevant results and screening full texts to identify excluded and included studies. Another author H.Y. would discuss together if there were any difference between two independent investigators and then came to an identical consensus. We also checked the references of reviewed articles and original researches to identify additional eligible trials. The reference lists of review articles and included studies were manually searched to locate articles that were not identified in the database search.

\section{Selection Criteria}

To be included in the selection, studies were required to present the following: (1) RCTs and observational studies (cohort studies and case-control studies) that evaluated pharmacist management of oral anticoagulation therapy; (2) included patients were diagnosed with the indications of warfarin; (3) the comparison was between pharmacist-led anticoagulation management and other management models that provided by physicians, nurses or other health care givers; (4) outcomes including either INR control status, haemorrhage events, thrombosis events, mortality, patient satisfaction or medicine cost saving.

Studies were excluded with the following criteria: (1) study types were not RCT, cohort studies or case-control studies.; (2) including patients without any indication of warfarin; (3) no comparison group and any comparison group data came from former studies or reports; (4) outcomes were not related to our study.

\section{Quality assessment of included studies}

Included RCTs were assessed the risk of bias according to the Cochrane Collaboration's tool from Cochrane Handbook and observational studies were assessed by Newcastle-Ottawa Scale (NOS) $(18,19)$, The methodological quality was assessed by two independent authors K.L.H. and X.L.C. and 
discrepancies were resolved by other authors (Z.K.Y. and L.H.L.).

\section{Quality of evidence}

Evidence from RCTs and observational studies of different outcomes were rated by the grading of recommendations assessment, development, and evaluation (GRADE) (20), which were finished by K.L.H. and Y.W. independently. And results were explained modestly referred to evidence grades.

\section{Outcomes}

The primary outcome was INR control condition, including the percentage of time within the target therapeutic range (TTR) and the percentage of time within the expanded therapeutic range (TER). Haemorrhage events, thrombosis events, mortality, patient satisfaction and cost savings were evaluated as secondary outcomes.

\section{Statistical analysis}

Two authors K.L.H and X.L.C finished data extraction independently after reading full texts of included studies and another author L.H.L discuss together if there's any disagreement. Dichotomous data were presented difference as odds ratio (OR) and $95 \%$ confidence interval $(\mathrm{CI})$, weight mean difference (WMD) and $95 \%$ CI presented continuous data. Heterogeneity analysis were performed by $\mathrm{I}^{2}$ statistic. $\mathrm{I}^{2}<25 \%$ indicated low heterogeneity, $\mathrm{I}^{2} \quad 25 \%-50 \%$ indicated moderate heterogeneity and $\mathrm{I}^{2}>50 \%$ indicated a significant heterogeneity (21). Random-effects modeling was used to calculate pooled WMDs/ORs and $95 \%$ CIs when $\mathrm{I}^{2}>50 \%$, fixed-effects modeling was used when $\mathrm{I}^{2} \leq 50 \%$. $p$-values of all the outcomes were two-tailed and $p<0.05$ was considered to be statistical significance. The sensitivity analysis was performed by exclusion of each study one by one and publication bias was estimated by Egger's test and Begg's test. Data pooled analysis, sensitivity analysis and publication bias analysis implemented by STATA (version 12.0, StataCorp, College Station). Quality of evidence assessment according to GRADE system performed by GRADEprofiler software (version3.6, Grade Working Group).

\section{RESULTS}

\section{Study selection}

4245 articles were initially searched for this analysis in PubMed, Embase, Cochrane Library, ClinicalTrials.gov and reference lists of yielded results. Finally, 16 studies satisfied our inclusion criteria (11-15, 22-34), including 8 RCTs $(14,15,22-$ 27) and 9 cohort observational studies $(11,12,28$ 34). Studies that only abstract accessible with incomplete data and not separate pharmacist-led treatment as an independent group were excluded. The full literature search process was shown in Figure 1 and characteristic of included studies were shown in Table 1. Other management models of included studies were provided by physicians in general hospitals, anticoagulation clinics and family medicine clinics.

\section{Quality assessment}

The Cochrane Collaboration's tool for assessing risk of bias graded on the selection bias (random sequence generation, allocation concealment), performance bias (blinding of participants and personnel), detection bias (blinding of outcome assessment), attrition bias (incomplete outcome data), reporting bias (selective reporting) and other bias (other sources of bias) with "low risk of bias", "high risk of bias" and "unclear risk of bias" (Figure 2). The NOS that assessed cohort studies is a "star system" that graded on selection, comparability, exposure or outcome and included studies were of high quality (Table 2).

\section{Quality of evidence}

We adopted the grading of recommendations assessment, development, and evaluation (GRADE) methodology to rate quality of the evidence from our meta-analysis results as high, moderate, low or very low. Evidence of RCTs begin as high quality and observational studies as low quality. Evidence may be decreased for following reasons: study limitations (risk of bias), inconsistency of results, indirectness of evidence, imprecision and publication bias. And evidence may rate up the quality for the large magnitude of effect, dose-response gradient and all plausible confounders or other biases increase our confidence in the estimated effect (20) (Table 3).

\section{INR control}

The primary outcome, TTR was reported in 6 studies (14, 15, 22, 28, 29, 31), including 3 RCTs $(14,15$, $22)$, and 3 cohort studies $(28,29,31)$. The target therapeutic range was defined as INR from 2.0 to 4.0 for different indications in the included studies. TTR was shown greater in pharmacist management group than the other management models in the pooled results (WMD: $8.03,95 \% \mathrm{CI}: 2.19-13.88, p=0.007$ ) and results from cohort studies were also statistical significance (WMD: 13.52, 95\%CI: 8.38-18.66, $p=0.000$ ). Whereas, results were also not significant from RCTs (WMD: 1.25, 95\%CI: -2.82-5.32, $p=0.548)$. Analysis of heterogeneity was considered a high level $\left(\mathrm{Chi}^{2}=24.89, p=0.000, \mathrm{I}^{2}=75.9 \%\right)$ of overall studies and results of RCTs and observational cohort studies had low to moderate heterogeneity 
$\left(\mathrm{Chi}^{2}=1.50, p=0.474, \mathrm{I}^{2}=0.0 \% ; \mathrm{Chi}^{2}=5.02, p=0.170\right.$, $\mathrm{I}^{2}=40.2 \%$ ) (Figure $3 \mathrm{~A}$ ). There was no obvious asymmetry found from Egger's test $(p=0.974)$ and Begg's test $(p=0.764)$ and the sensitivity analysis showed pooled results were reliable. We rated the quality of evidence from RCTs results as moderate due to serious risk of bias and observational studies results as low-quality.

The expanded target range was defined as target therapeutic INR range \pm 0.2 mostly, while Verret et al defined it as target therapeutic INR range \pm 0.3 (15). TER was not significantly improved in pharmacist management group in the pooled results from 3 RCTs $(14,15,22)$, and one cohort study (31), (WMD: 4.07, 95\%CI: $-1.75-9.89, p=0.170)$. There was a high heterogeneity among overall included studies $\left(\mathrm{Chi}^{2}=16.57, p=0.001, \mathrm{I}^{2}=81.9 \%\right)$. Similarly, results from RCTs were not statistical significant (WMD: 1.35, 95\%CI: -1.12-3.82, $p=0.285)$ with low heterogeneity $\left(\mathrm{Chi}^{2}=0.75, p=0.687, \mathrm{I}^{2}=0.0 \%\right.$ (Figure
3B). The publication bias analysis by Egger's test showed no statistically significant with the $p$-value of 0.670 and Begg's test with the $p$-value of 0.734 . The analysis of sensitivity indicated that the results was reliable. Using GRADE, we rated quality of evidence of RCTs results as moderate-quality.

The percentage of time within the target therapeutic range and the percentage of time within the expanded therapeutic range were calculated using a linear interpolation between INR values that established by Rosendaal et al (35).

Furthermore, INR value above and below the therapeutic range were reported in several studies. Patients of pharmacist management group spent less time in $\mathrm{INR}<2.0$ ( $1.6 \pm 0.8$ days vs $1.8 \pm 1.1$ days, $p=0.61$ ) of Airee et al (28). Whereas, different results from Verret et al and Lalonde et al studies showed the percentage of time outside critical INR value (INR $<1.5$ or INR $>5$. 0 ) were slightly higher in pharmacist management group (14), (15).

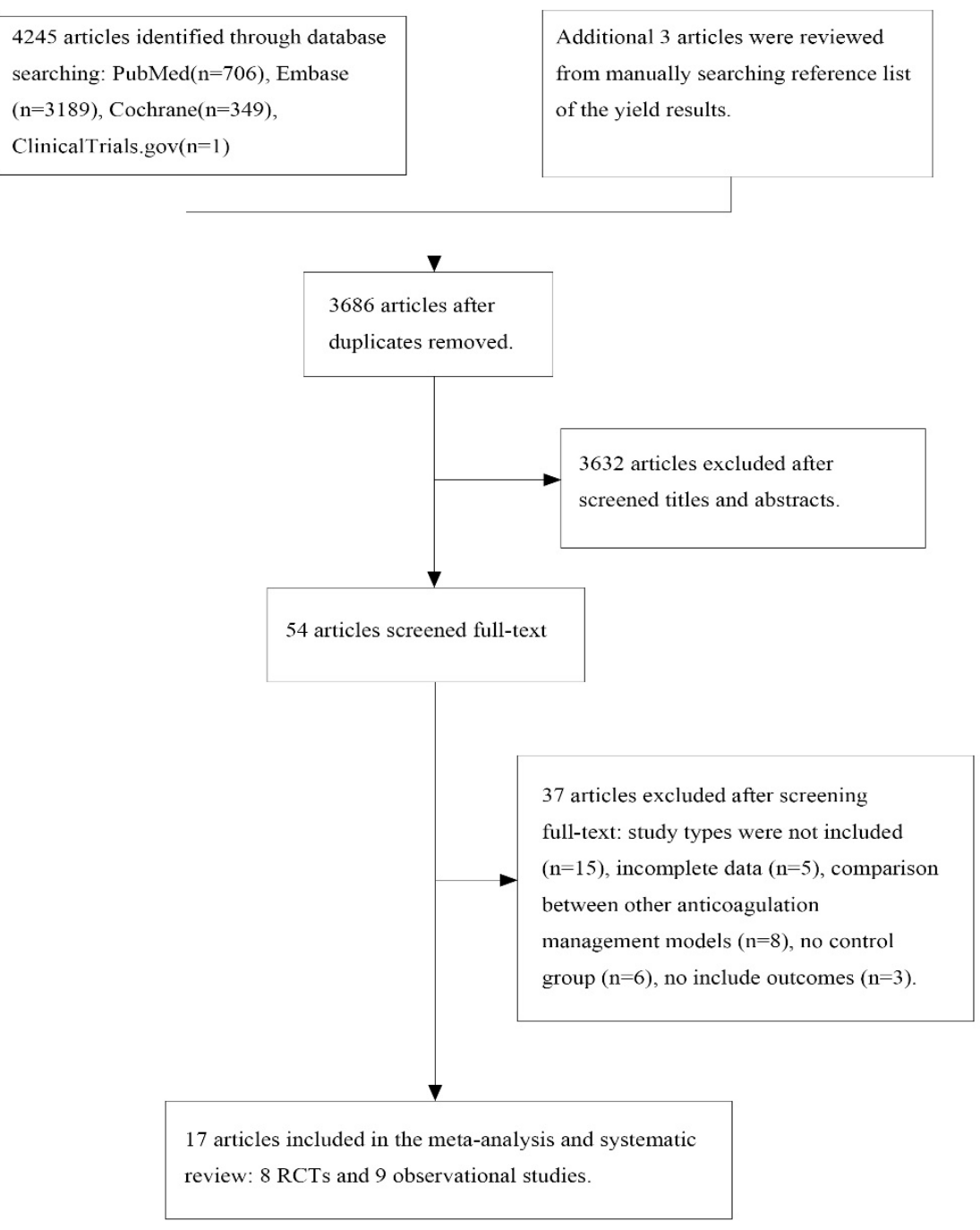

Figure 1. Flow chart of the selection process of included studies. 
J Pharm Pharm Sci (www.cspsCanada.org) 20, 378 - 396, 2017

Table 1. Characteristic of included studies

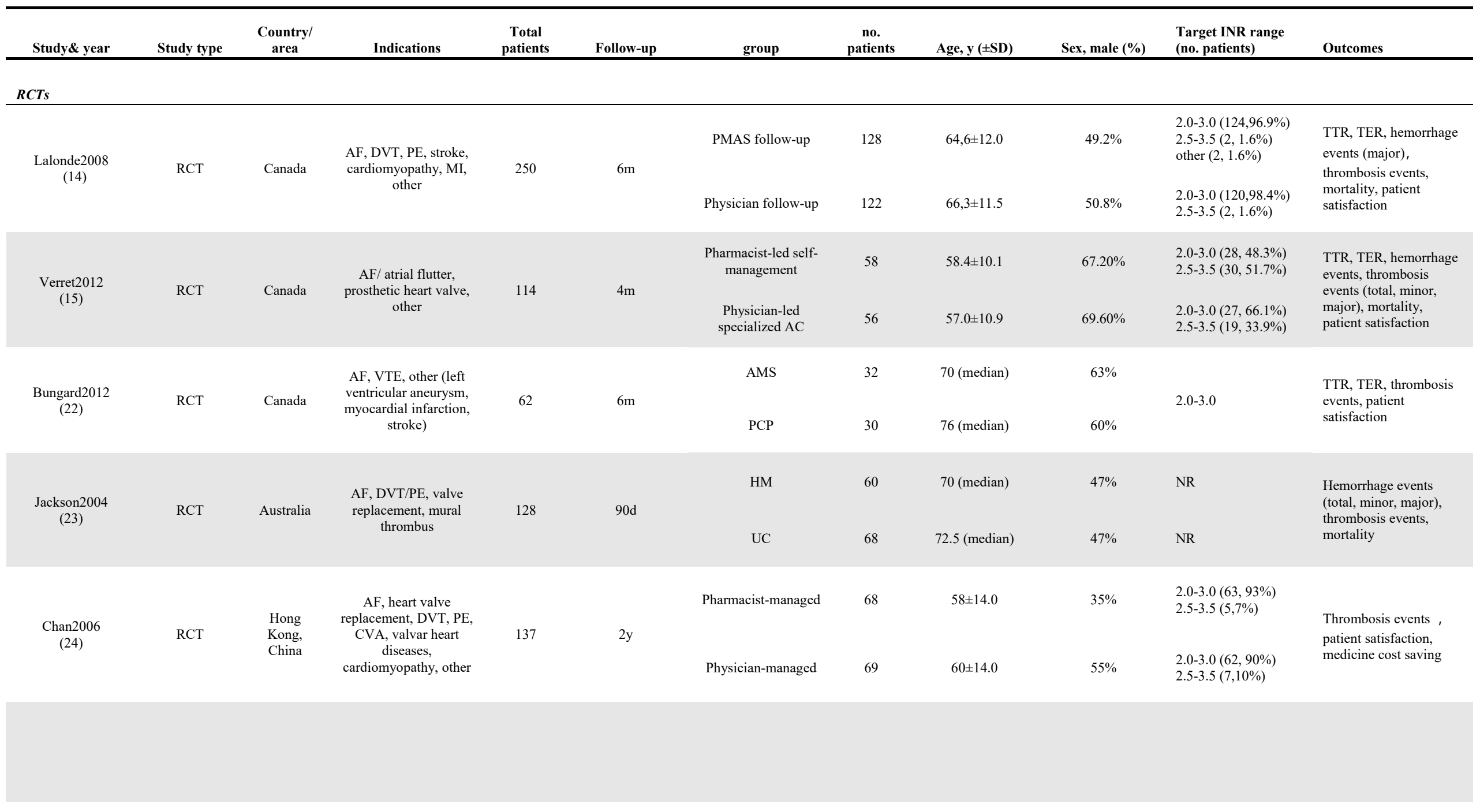


J Pharm Pharm Sci (www.cspsCanada.org) 20, 378 - 396, 2017

Table 1 Continued...

\begin{tabular}{|c|c|c|c|c|c|c|c|c|c|c|c|}
\hline $\begin{array}{l}\text { Schillg2011 } \\
\text { (25) }\end{array}$ & RCT & America & VTE, AF & 500 & $30 \mathrm{~d}$ & PDAS & 250 & $64.1 \pm 15.6$ & $54.0 \%$ & NR & $\begin{array}{l}\text { Hemorrhage events } \\
\text { (major), thrombosis } \\
\text { events }\end{array}$ \\
\hline $\begin{array}{l}\text { Wilson2003 } \\
\text { (26) }\end{array}$ & RCT & Canada & $\begin{array}{l}\text { VTE, AF, mechanical } \\
\text { heart valve }\end{array}$ & 221 & $3 \mathrm{~m}$ & $\mathrm{AC}$ & 112 & $61 \pm 15$ & $62 \%$ & $\begin{array}{l}2.0-3.0(101,90 \%) \\
2.5-3.5(9,8 \%) \\
3.0-4.0(2,2 \%) \\
\\
2.0-3.0(98,90 \%) \\
2.5-3.5(10,9 \%) \\
1.5-2.0(1,1 \%)\end{array}$ & $\begin{array}{l}\text { Hemorrhage events } \\
\text { (major), thrombosis } \\
\text { events, mortality }\end{array}$ \\
\hline $\begin{array}{l}\text { Lakshim2013 } \\
\text { (27) }\end{array}$ & RCT & India & $\begin{array}{l}\text { Mitral valve } \\
\text { replacement, AF, DVT, } \\
\text { PE, valvotomy, } \\
\text { bioprosthetic valve, } \\
\text { other. }\end{array}$ & 80 & $6 \mathrm{~m}$ & AMS & 40 & 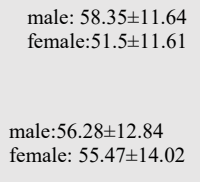 & $60 \%$ & $\begin{array}{l}2.0-3.0(77.5 \%) \\
2.5-3.5(22.5 \% \\
\\
2.0-3.0(101,90 \%) \\
2.5-3.5(9,8 \%) \\
3.0-4.0(2,2 \%)\end{array}$ & $\begin{array}{l}\text { Hemorrhage events } \\
\text { (minor, major), } \\
\text { thrombosis events }\end{array}$ \\
\hline
\end{tabular}

Observational studies

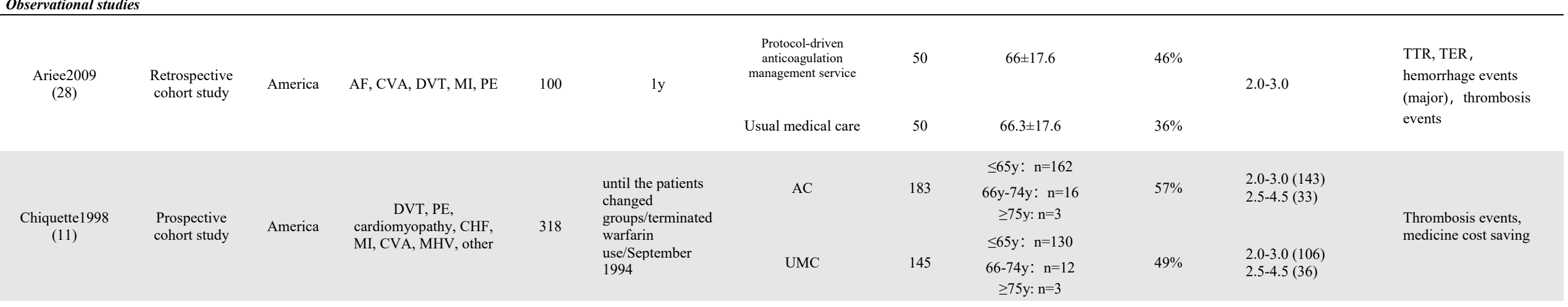




\section{Table 1 Continued...}

\begin{tabular}{|c|c|c|c|c|c|c|c|c|c|c|c|}
\hline $\begin{array}{l}\text { Elewa2016 } \\
\quad(29)\end{array}$ & $\begin{array}{l}\text { Retrospective } \\
\text { cohort study }\end{array}$ & Qatar & $\begin{array}{l}\text { AF, valve replacement, } \\
\text { DVT/PE, other }\end{array}$ & 278 & $6 \mathrm{~m}-1 \mathrm{y}$ & $\begin{array}{l}\text { Pharmacist-based } \\
\text { clinic } \\
\text { Doctor-based clinic }\end{array}$ & 200 & $65.9 \pm 10.9$ & $53.8 \%$ & $\begin{array}{l}2.0-3.0(67,85.9 \%) \\
2.5-3.5(11,14.1 \%) \\
\\
2.0-3.0(193,96.5 \%) \\
2.5-3.5(7,7.5 \%)\end{array}$ & TTR \\
\hline $\begin{array}{l}\text { Sargent2016 } \\
\quad(30)\end{array}$ & $\begin{array}{l}\text { Retrospective } \\
\text { cohort study }\end{array}$ & Canada & AF, DVT, PE & 197 & $1 \mathrm{y}$ & $\begin{array}{l}\text { Pharmacist-managed } \\
\text { warfarin-dosing } \\
\text { Physician-managed } \\
\text { warfarin-dosing }\end{array}$ & 116 & $\begin{array}{c}\text { site } 1: 82 \\
\text { site2:85 } \\
\\
\quad 83\end{array}$ & $\begin{array}{l}\text { site1: } 22.2 \% \\
\text { site2: } 7 \% \\
\\
\\
24.7 \%\end{array}$ & $2.0-3.0$ & $\begin{array}{l}\text { Hemorrhage events } \\
\text { (major) }\end{array}$ \\
\hline $\begin{array}{l}\text { Thanimalai2013 } \\
\text { (31) }\end{array}$ & $\begin{array}{l}\text { Retrospective } \\
\text { cohort study }\end{array}$ & Malaysia & $\begin{array}{l}\text { AF, MVR, aortic valve } \\
\text { replacement, DVT, } \\
\text { others }\end{array}$ & 184 & $6 \mathrm{~m}$ & WMTAC & 92 & $63,2 \pm 13.0$ & $50.0 \%$ & $\begin{array}{l}2.0-3.0 \text { for } \\
\text { prophylaxis and for } \\
\text { the treatment of } \\
\text { uncomplicated } \\
\text { disease } \\
2.5-3.5 \text { for patients } \\
\text { with MHV or for } \\
\text { failure with previous } \\
\text { warfarin treatment }\end{array}$ & $\begin{array}{l}\text { TTR, TER, hemorrhage } \\
\text { events (total, major) }\end{array}$ \\
\hline $\begin{array}{l}\text { Witt2005 } \\
\text { (12) }\end{array}$ & $\begin{array}{l}\text { Retrospective } \\
\text { cohort study }\end{array}$ & America & $\begin{array}{c}\mathrm{AF}, \mathrm{CVA} / \text { stroke, } \\
\mathrm{DVT} / \mathrm{PE}, \text { arterial } \\
\text { thromboembolism, } \\
\text { prosthetic heart valve, } \\
\text { cardiomyopathy, } \\
\text { coronary artery disease }\end{array}$ & 6645 & $6 \mathrm{~m}$ & $\begin{array}{l}\text { CPAS } \\
\text { UC }\end{array}$ & 3323 & $\begin{array}{l}67.5 \pm 13.3 \\
68.1 \pm 12.6\end{array}$ & $\begin{array}{l}52.0 \% \\
54.4 \%\end{array}$ & $\begin{array}{l}2.0-3.0(2641,79.5 \%) \\
2.5-3.5(381,11.5 \%) \\
\text { other }(300,9.0 \%) \\
\\
2.0-3.0(2612,78.6 \%) \\
2.5-3.5(36511.0 \%) \\
\text { other }(345,10.4 \%)\end{array}$ & $\begin{array}{l}\text { Hemorrhage events } \\
\text { (major), thrombosis } \\
\text { events, mortality }\end{array}$ \\
\hline $\begin{array}{l}\text { Young2011 } \\
\quad(32)\end{array}$ & $\begin{array}{l}\text { Retrospective } \\
\text { cohort study }\end{array}$ & Canada & $\begin{array}{c}\text { AF, MHV, DVT, PE, } \\
\text { CVA, MI and/or ACS, } \\
\text { other }\end{array}$ & 193 & $17 \mathrm{~m}$ & $\mathrm{PC}$ & 112 & $67 \pm 18$ & $51 \%$ & $\begin{array}{l}2.0-3.0(93,83 \%) \\
2.5-3.5(19,17 \%) \\
\\
2.0-3.0(65,80 \%) \\
2.5-3.5(16,20 \%)\end{array}$ & $\begin{array}{l}\text { hemorrhage events } \\
\text { (major) }\end{array}$ \\
\hline
\end{tabular}


Table 1 Continued...

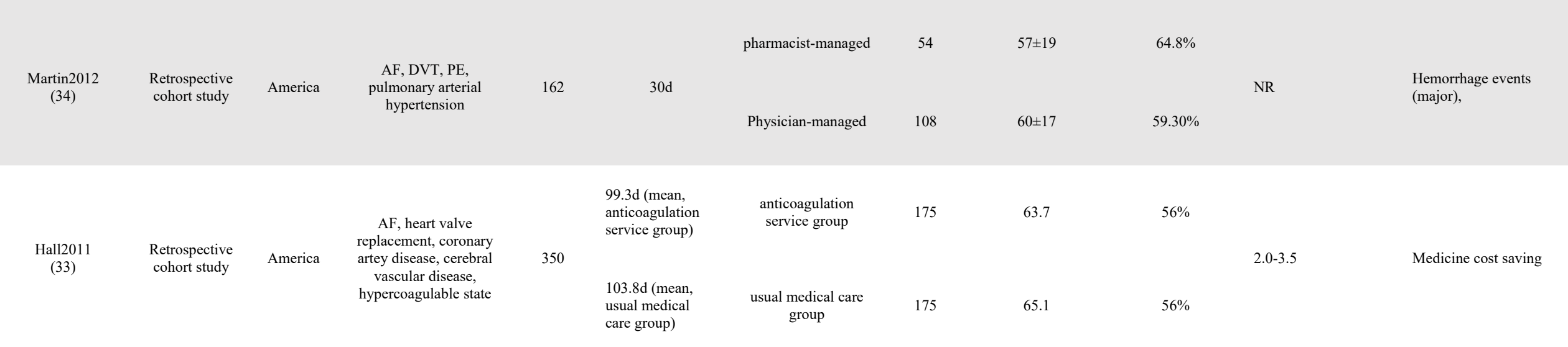

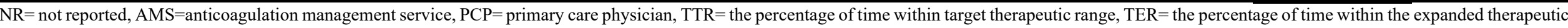

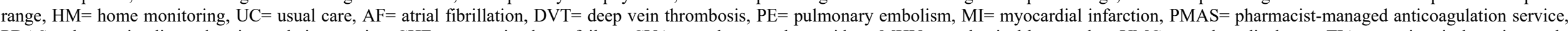

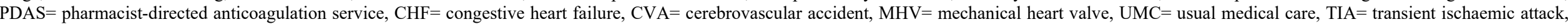

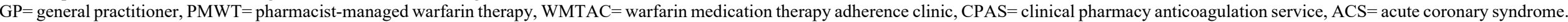

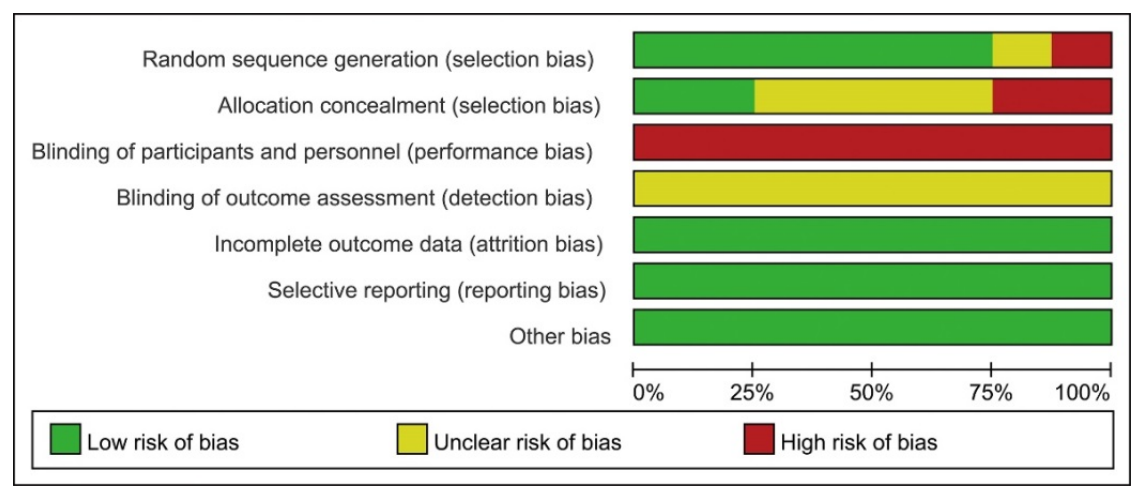

Figure 2. Risk of bias assessments on the eligible RCTs. 


\section{Haemorrhage events}

Total haemorrhage events were reported in 3 RCTs $(15,23,27)$ and 3 cohort studies $(11,28,31)$, pharmacist-led management group undergone less haemorrhage events than control group (OR: 0.43, 95\% CI: $0.23-0.78, \quad p=0.006)$ with high heterogeneity $\left(\mathrm{Chi}^{2}=12.45, p=0.029, \mathrm{I}^{2}=59.8 \%\right)$ of overall result. Pooled results from observational studies showed significantly decreased in haemorrhage events of pharmacist-led group (OR: $0.30,95 \%$ CI: $0.19-0.47, p=0.000 ; \mathrm{Chi}^{2}=1.83$, $p=0.400, \mathrm{I}^{2}=0.0 \%$ ), but results from RCTs showed no significant differences between two groups (OR: $0.47, \quad 95 \%$ CI: $0.17-1.28, \quad p=0.140)$. High heterogeneity verified in RCTs results $\left(\mathrm{Chi}^{2}=7.80\right.$, $\left.p=0.02, \mathrm{I}^{2}=74.4 \%\right)$ and low heterogeneity was detected in observational studies $\left(\mathrm{Chi}^{2}=1.83\right.$, $p=0.400, \mathrm{I}^{2}=0.0 \%$ ) (Figure 4A). Reliability of the pooled results was certified by sensitivity analysis. The publication bias analysis by Egger's test and Begg's test showed no statistically significant ( $p=0.619, p=0.707)$. We downgraded RCTs results as low-quality evidence due to risk of bias and imprecision and upgraded evidence from observational studies as moderate-quality for the large magnitude of effect.

Three RCTs $(15,23,27)$ and 3 observational cohort studies $(11,28,31)$, reported minor haemorrhage events, which the pooled results exposed significant difference between pharmacistled management group and control group (OR: 0.52, 95\% CI: $0.30-0.90, \quad p=0.020) \quad$ with high heterogeneity $\left(\mathrm{Chi}^{2}=10.15, p=0.071, \mathrm{I}^{2}=50.7 \%\right)$ and observational studies pooled results were similar (OR: $0.33,95 \%$ CI: 0.18-0.61, $p=0.000$ ). RCTs pooled results showed no significant decrease of minor haemorrhage events in pharmacist-led management group (OR: 0.65, 95\% CI: 0.35-1.19, $p=0.162)$ and heterogeneity analysis detected moderate heterogeneity $\left(\mathrm{Chi}^{2}=3.05, \quad p=0.218\right.$, $\mathrm{I}^{2}=34.3 \%$ ) (Figure 4B). There was no substantial modification in the sensitivity analysis and Egger's test $(p=0.299)$ and Begg's test $(p=0.707)$ showed no publication bias. Quality of evidence from RCTs results was rated as low due to risk of bias and moderate-quality evidence of observational studies for the large magnitude of effect. For the major haemorrhage events, pooled results from 7 RCTs (14, $15,23-27)$, and 7 cohort studies $(11,12,28,30-32$, 34 ), indicated that no significant difference of pharmacist-led management in major haemorrhage events (OR: $0.76,95 \%$ CI: 0.53-1.10, $p=0.143$ ) with a low homogeneity $\left(\mathrm{Chi}^{2}=11.46, p=0.572, \mathrm{I}^{2}=0.0 \%\right)$.
Pooled results of RCTs and cohort studies came to a similar result with overall studies (RCTs: OR: 0.65, 95\%CI: $0.32-1.33, p=0.237$; observational studies: OR: 0.81, 95\%CI: 0.53-1.23, $p=0.317$ ) (Figure 4C). Major haemorrhage events was defined as "major bleeding" of the International Society of Thrombosis and Hemostasis (ISTH) which including fatal bleeding in non-surgical patients, symptomatic bleeding, bleeding causing a fall in hemoglobin level of $20 \mathrm{~g} / \mathrm{L}$, or need for transfusion (36). The publication bias was not found from Egger's test $(p=0.864)$ and Begg's test $(p=0.584)$. The sensitivity analysis indicated robustness of the results. According to GRADE, we downgraded evidence of RCTs results as low-quality and we downgraded evidence of observational studies as very-low-quality because of imprecision.

\section{Thrombosis events}

Seven RCTs $(14,15,22-26)$, and 3 cohort studies $(11,12,28)$, compared pharmacist-led management model with other models and overall results showed that pharmacist-led management significantly decreased the risk of thrombosis events (OR: 0.46, 95\%CI: $0.30-0.70, p=0.000$ ) and pooled results of cohort studies were consistent with overall results (OR: 0.38. 95\%CI: 0.23-0.63, $p=0.000$ ). However, results of RCTs showed no statistical significance (OR: 0.80, 95\%CI: 0.34-1.88, $p=0.615$ ) (Figure 5). Overall, RCTs and observational studies pooled results were low heterogeneity showed in (overall: $\mathrm{Chi}^{2}=3.28, p=0.952, \mathrm{I}^{2}=0.0 \%$; RCTs: $\mathrm{Chi}^{2}=0.26$, $p=1.000, \mathrm{I}^{2}=0.0 \%$; observational studies: $\mathrm{Chi}^{2}=0.79$, $\left.p=0.672, \mathrm{I}^{2}=0.0 \%\right)$. Thrombosis events were reported including deep vein thrombosis (DVT), pulmonary embolism (PE), stroke, transient infarction attack (TIA) and myocardial infarction (MI), et al. No publication bias indicated in Egger's test $(p=0.169)$ and Begg's test $(p=0.536)$. The sensitivity analysis showed the pooled results were reliable. Evidence of RCTs results was rated as lowquality due to risk of bias and imprecision and evidence of observational studies results was upgraded for the large magnitude of effect to moderate-quality.

\section{Mortality}

Five studies $(12,14,15,23,26)$, reported patient death in follow-up period, including 4 RCTs $(14,15$, $23,26)$, and one cohort study (12). There was no significant reduction of mortality in pharmacist-led 
J Pharm Pharm Sci (www.cspsCanada.org) 20, 378 - 396, 2017

\begin{tabular}{|c|c|c|c|c|c|c|c|c|c|}
\hline \multirow[b]{2}{*}{ Reference } & \multicolumn{4}{|c|}{ Selection } & \multirow{2}{*}{$\begin{array}{l}\text { Compa- } \\
\text { rability }\end{array}$} & \multicolumn{4}{|c|}{ Outcomes } \\
\hline & Exposed cohort & $\begin{array}{l}\text { Nonexpos- } \\
\text { ed cohort }\end{array}$ & $\begin{array}{c}\text { Exposure ascer- } \\
\text { tainment }\end{array}$ & $\begin{array}{l}\text { Interest } \\
\text { outcome }\end{array}$ & & Assessment & $\begin{array}{c}\text { Follow up } \\
\text { length }\end{array}$ & $\begin{array}{l}\text { Follow up } \\
\text { adequacy }\end{array}$ & Total \\
\hline (28) & * & * & * & * & $* *$ & * & * & & 8 \\
\hline (29) & $*$ & $*$ & $*$ & $*$ & $* *$ & $*$ & $*$ & $*$ & 9 \\
\hline (20) & * & * & * & * & $* *$ & * & * & * & 9 \\
\hline (31) & $*$ & $*$ & $*$ & $*$ & $* *$ & $*$ & $*$ & & 8 \\
\hline (12) & * & * & * & * & $* *$ & * & * & $*$ & 9 \\
\hline (32) & $*$ & $*$ & * & * & $* *$ & $*$ & $*$ & & 8 \\
\hline (11) & * & $*$ & * & * & $* *$ & * & & $*$ & 8 \\
\hline (34) & $*$ & $*$ & $*$ & $*$ & $* *$ & $*$ & & & 7 \\
\hline (33) & * & * & * & & $* *$ & * & * & * & 8 \\
\hline
\end{tabular}

Table 3. Summary of findings of GRADE evidence

\begin{tabular}{|c|c|c|c|c|c|}
\hline \multirow[t]{3}{*}{ Outcomes } & \multicolumn{2}{|c|}{ Illustrative comparative risks* $(95 \% \mathrm{CI})$} & \multirow{3}{*}{$\begin{array}{l}\text { Relative effect } \\
95 \% \text { CI }\end{array}$} & \multirow{3}{*}{$\begin{array}{l}\text { No of Participants } \\
\text { and studies }\end{array}$} & \multirow{3}{*}{$\begin{array}{l}\text { Quality of evidence } \\
\text { GRADE }\end{array}$} \\
\hline & Assumed risk & Corresponding risk & & & \\
\hline & Control & Pharmacist-led & & & \\
\hline Percentage of time within the target therapeutic & & WMD 1.25 & & 426 & $\oplus \oplus \oplus \ominus$ \\
\hline range-RCT; Follow-up: 4 to 6 months & & $(-2.82$ to 5.32$)$ & & (3 studies) & moderate $^{1}$ \\
\hline Percentage of time within the expanded therapeutic & & WMD 1.35 & & 426 & $\oplus \oplus \oplus \ominus$ \\
\hline range-RCT; Follow-up: 4 to 6 months & & $(-1.12$ to 3.82$)$ & & (3 studies) & moderate $^{1}$ \\
\hline Total haemorrhage events-RCT & 395 per 1000 & 292 per 1000 & OR 0.63 & 241 & $\oplus \oplus \ominus \ominus$ \\
\hline Follow-up: 3 to 4 months & & $(195$ to 414$)$ & $(0.37$ to 1.08$)$ & (2 studies) & low $^{1,2}$ \\
\hline
\end{tabular}


J Pharm Pharm Sci (www.cspsCanada.org) 20, 378 - 396, 2017

\section{Table 3 Continued...}

\begin{tabular}{|c|c|c|c|c|c|}
\hline $\begin{array}{l}\text { Minor haemorrhage events-RCT } \\
\text { Follow-up: } 3 \text { to } 6 \text { months }\end{array}$ & 415 per 1000 & $\begin{array}{l}319 \text { per } 1000 \\
(225 \text { to } 431)\end{array}$ & $\begin{array}{l}\text { OR } 0.66 \\
(0.41 \text { to } 1.07)\end{array}$ & $\begin{array}{l}321 \\
\text { (3 studies) }\end{array}$ & $\begin{array}{l}\oplus \oplus \Theta \Theta \\
\text { low }^{1,3}\end{array}$ \\
\hline $\begin{array}{l}\text { Major haemorrhage events-RCT } \\
\text { Follow-up: } 1 \text { to } 24 \text { months }\end{array}$ & 28 per 1000 & $\begin{array}{l}18 \text { per } 1000 \\
(9 \text { to } 37)\end{array}$ & $\begin{array}{l}\text { OR } 0.65 \\
(0.32 \text { to } 1.33)\end{array}$ & $\begin{array}{l}1289 \\
\text { ( } 7 \text { studies) }\end{array}$ & $\begin{array}{l}\oplus \oplus \ominus \ominus \\
\text { low }^{1,4}\end{array}$ \\
\hline $\begin{array}{l}\text { Thrombosis events-RCT } \\
\text { Follow-up: } 1 \text { to } 24 \text { months }\end{array}$ & 17 per 1000 & $\begin{array}{l}14 \text { per } 1000 \\
(6 \text { to } 32)\end{array}$ & $\begin{array}{l}\text { OR } 0.80 \\
(0.34 \text { to } 1.88)\end{array}$ & $\begin{array}{l}1408 \\
\text { (7 studies) }\end{array}$ & $\begin{array}{l}\oplus \oplus \Theta \Theta \\
\text { low }^{1,5}\end{array}$ \\
\hline $\begin{array}{l}\text { Mortality-RCT } \\
\text { Follow-up: } 3 \text { to } 6 \text { months }\end{array}$ & 31 per 1000 & $\begin{array}{l}29 \text { per } 1000 \\
(13 \text { to } 65)\end{array}$ & $\begin{array}{l}\text { OR } 0.94 \\
(0.4 \text { to } 2.17)\end{array}$ & $\begin{array}{l}709 \\
\text { (4 studies) }\end{array}$ & $\begin{array}{l}\oplus \oplus \ominus \ominus \\
\text { low }^{1,6}\end{array}$ \\
\hline $\begin{array}{l}\text { Percentage of time within the target therapeutic } \\
\text { range-OS }\end{array}$ & & $\begin{array}{l}\text { WMD } 13.52 \\
(8.38 \text { to } 18.66)\end{array}$ & & $\begin{array}{l}562 \\
\text { (3 studies) }\end{array}$ & $\begin{array}{l}\oplus \oplus \Theta \Theta \\
\text { low }^{1}\end{array}$ \\
\hline \multicolumn{6}{|l|}{ Follow-up: 6 to 12 months } \\
\hline $\begin{array}{l}\text { Total haemorrhage events-OS } \\
\text { Follow-up: } 6 \text { to } 12 \text { months }\end{array}$ & 278 per 1000 & $\begin{array}{l}104 \text { per } 1000 \\
(68 \text { to } 153)\end{array}$ & $\begin{array}{l}\text { OR } 0.30 \\
(0.19 \text { to } 0.47)\end{array}$ & $\begin{array}{l}602 \\
\text { (3 studies) }\end{array}$ & $\begin{array}{l}\oplus \oplus \oplus \ominus \\
\text { moderate }^{7,8}\end{array}$ \\
\hline $\begin{array}{l}\text { Minor haemorrhage events-OS } \\
\text { Follow-up: } 6 \text { to } 12 \text { months }\end{array}$ & 243 per 1000 & $\begin{array}{l}90 \text { per } 1000 \\
(57 \text { to } 138)\end{array}$ & $\begin{array}{l}\text { OR } 0.31 \\
(0.19 \text { to } 0.5)\end{array}$ & $\begin{array}{l}602 \\
\text { (3 studies) }\end{array}$ & $\begin{array}{l}\oplus \oplus \oplus \ominus \\
\text { moderate }^{8,9}\end{array}$ \\
\hline $\begin{array}{l}\text { Major haemorrhage events-OS } \\
\text { Follow-up: } 1 \text { to } 17 \text { months }\end{array}$ & 13 per 1000 & $\begin{array}{l}11 \text { per } 1000 \\
(7 \text { to } 16)\end{array}$ & $\begin{array}{l}\text { OR } 0.81 \\
(0.53 \text { to } 1.23)\end{array}$ & $\begin{array}{l}7809 \\
\text { (6 studies) }\end{array}$ & $\begin{array}{c}\oplus \ominus \ominus \ominus \\
\text { very low }{ }^{10} \\
\end{array}$ \\
\hline $\begin{array}{l}\text { Thrombosis events-OS } \\
\text { Follow-up: } 6 \text { to } 12 \text { months }\end{array}$ & 15 per 1000 & $\begin{array}{l}6 \text { per } 1000 \\
(4 \text { to } 10)\end{array}$ & $\begin{array}{l}\text { OR } 0.38 \\
(0.23 \text { to } 0.63)\end{array}$ & $\begin{array}{l}7063 \\
\text { (3 studies) }\end{array}$ & $\begin{array}{l}\oplus \oplus \oplus \ominus \\
\text { moderate }^{8,11}\end{array}$ \\
\hline
\end{tabular}

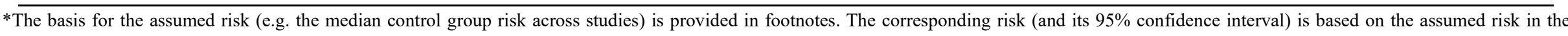

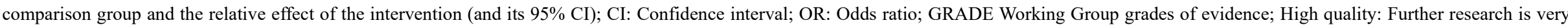

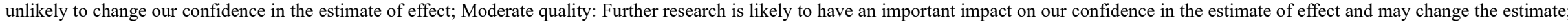

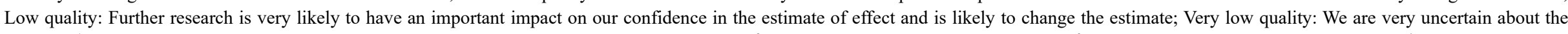

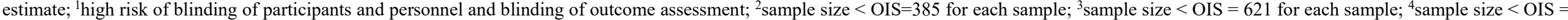

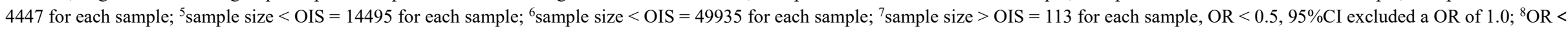

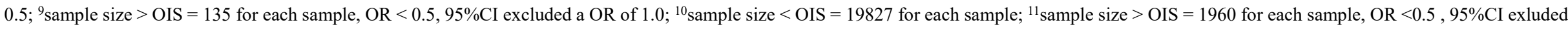
a OR of 1.0. 


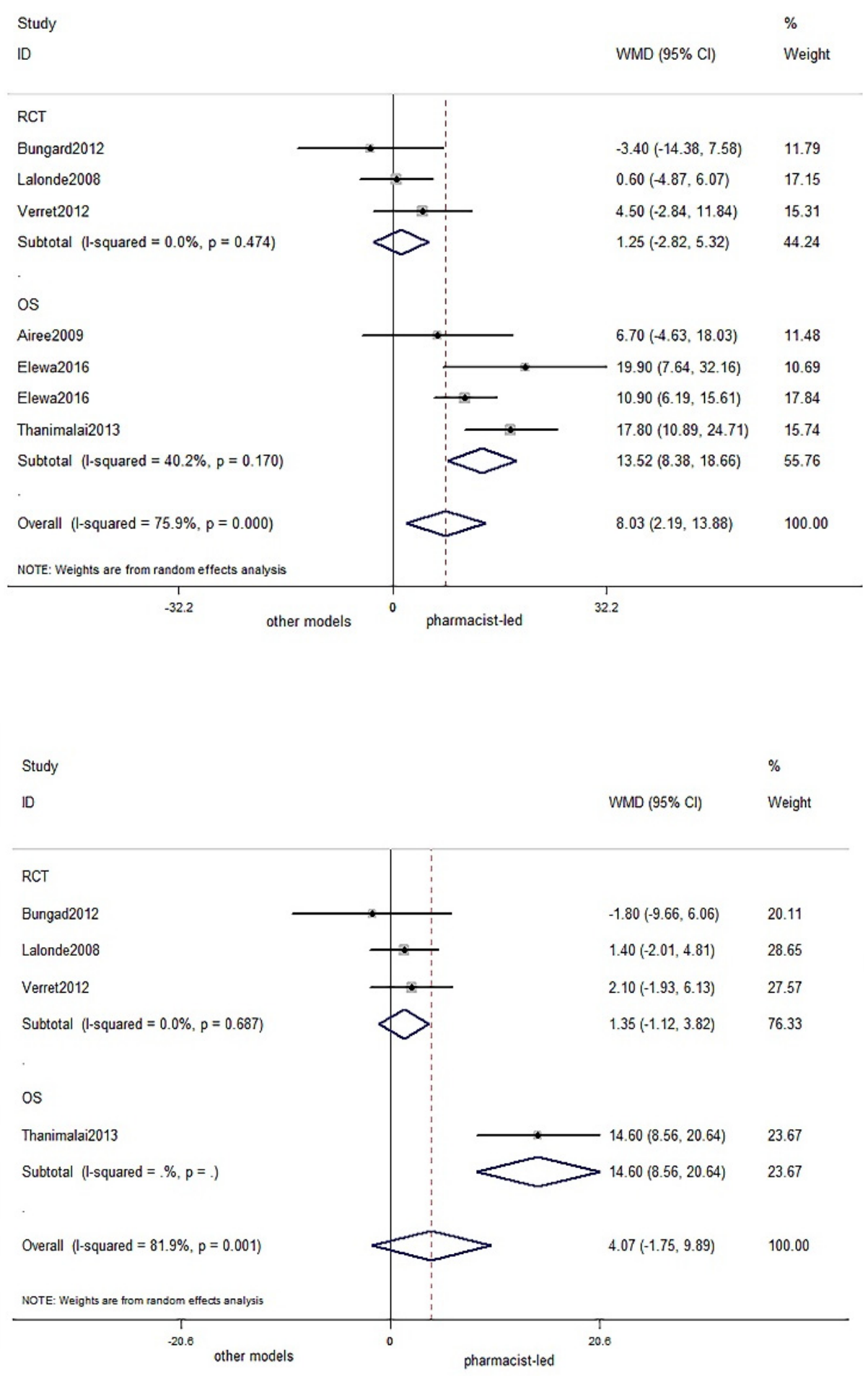

Figure 3. Percentage of time within the target therapeutic range (top) and within the expanded therapeutic range (bottom) between pharmacist-led and other models groups 

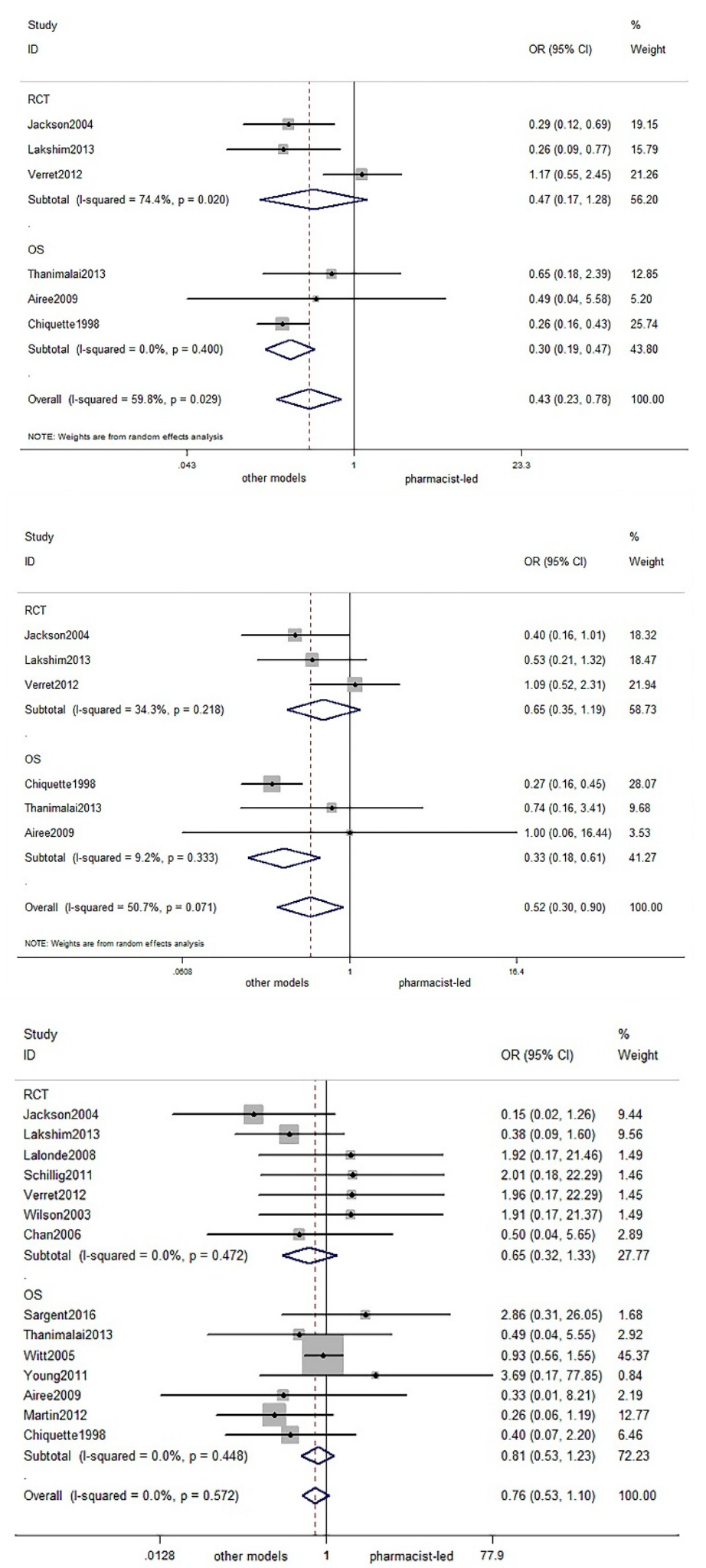

Figure 4. Total haemorrhage events (top), minor haemorrhage events (middle) and major haemorrhage events(bottom) between pharmacist-led management group and another models group. 


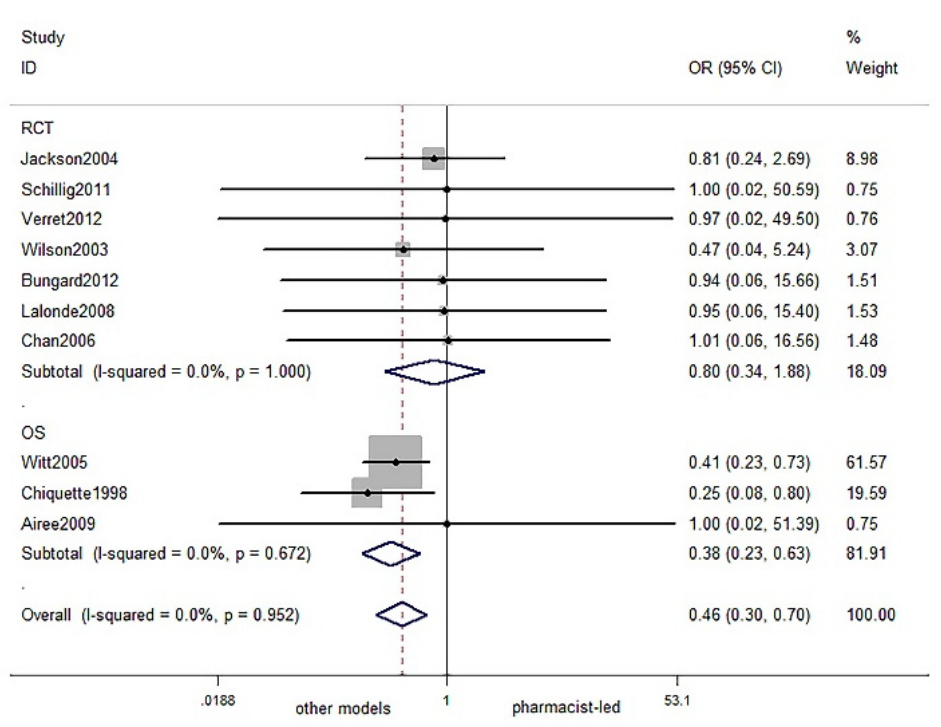

Figure 5. Thrombosis events between pharmacist-led management group and other models group.

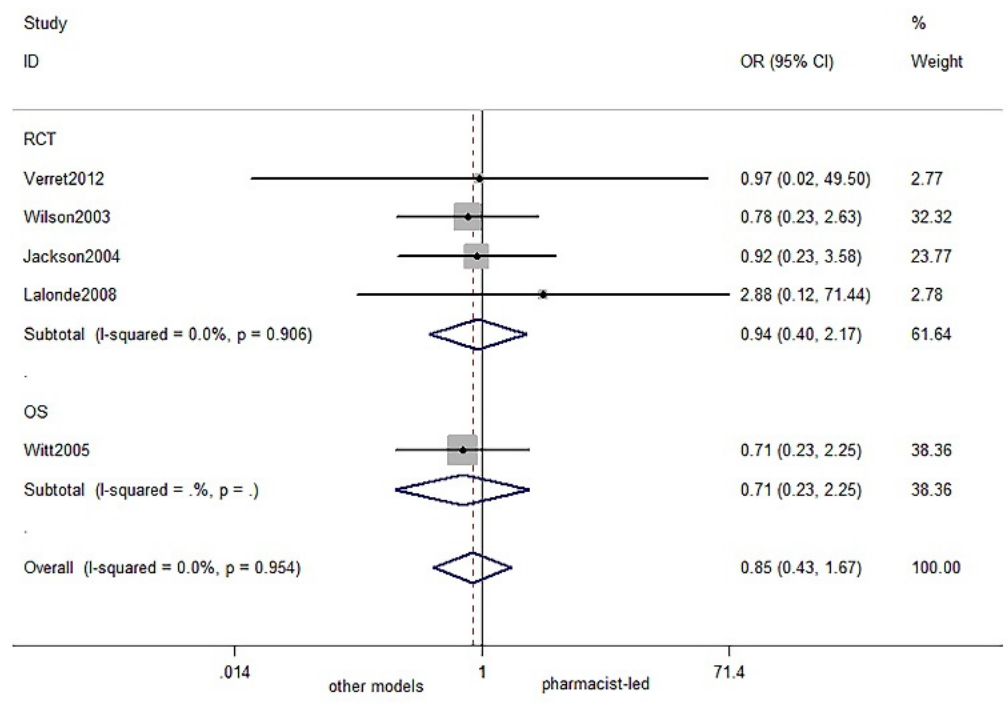

Figure 6. Forest plot of mortality between pharmacist-led management group and other models group.

management group (OR: 0.85 , 95\%CI: 0.43-1.67, $p=0.638)$ and the same results from RCTs pooled results (OR: $0.94,95 \% \mathrm{CI}: 0.40-2.17, p=0.876$ ) with low heterogeneity $\left(\mathrm{Chi}^{2}=0.68, p=0.954, \mathrm{I}^{2}=0.0 \%\right.$; $\mathrm{Chi}^{2}=0.56, p=0.906, \mathrm{I}^{2}=0.0 \%$, respectively) (Figure 6). Thanks to risk of bias and imprecision, evidence of RCTs results downgraded as low-quality. The publication bias was not found from Egger's test result $(p=0.155)$ and Begg's test result $(p=0.221)$ and the result was robust showed in the sensitivity analysis.

\section{Patient satisfaction}

4 RCTs $(14,15,22,24)$, reported patient satisfaction with different oral anticoagulation management models. The patient treatment satisfaction measured by 3 different surveys and questionnaires. Lalonde et al and Verret et al adopted a previously developed and validated questionnaire, which including 5 topics and one of them was general treatment satisfaction $(14,15,37)$. Verret et al reported that the change from baseline to end of study $(4.1 \pm 1.1$ vs $5.3 \pm 0.7, \quad p<0.001) \quad$ in pharmacist-led selfmanagement group and the general treatment 
satisfaction was also better than control group $(+1.3 \pm 1.2$ vs $+0.2 \pm 1.1, p<0.01)(15)$. Bungard et al adopted a validated survey instrument, the Duke Anticoagulation Satisfaction Scale (DASS) that assessed the overall satisfaction with treatment through 3 scales relevant to warfarin therapy $(22,38)$. The pharmacist-led AMS group had a significantly more satisfied with their anticoagulation management $(p=0.0001)$. The patient satisfaction questionnaire (PSQ-18) (RAND Corporation, Santa, Monica, CA, USA) was applied by Chan et al, which was a seven scales assessment including general satisfaction and similar results came from two groups. The overall score of pharmacist-managed group were significantly higher than that of physician-managed group (3.8 \pm 0.2 vs $3.6 \pm 0.3$, $p<0.001)(24)$.

\section{Medicine cost saving}

One RCT (24), and 2 observational cohort study (11, 33 ), reported cost of anticoagulation management in different currency units and time units. Chan et al indicated a significant reduction of the cost per patient per month in pharmacist management group ( $\$ 76 \pm 95$ vs $\$ 98 \pm 158, p<0.01,2006$ ) (24). Hall et al reported that the overall net medical care costs savings that accounting for anticoagulation service operational costs and available pharmacy expenditures was $\$ 3697$ per patient in the pharmacist-managed anticoagulation service group (33). The total cost contained anticoagulation therapy follow-up, hospitalizations and emergency room visits that related to warfarin use were lower in pharmacist management group ( $\$ 35326 / 100$ patientyears vs $\$ 167412 / 100$ patient-years, 1998) from Chiquette et al study (11).

\section{DISCUSSION}

We performed this meta-analysis including RCTs and observational studies and identified whether the pharmacist-led anticoagulation management was beneficial for patients compared to other models in INR control, haemorrhage events, thrombosis events, mortality, patient satisfaction and medicine cost saving. There were 17 studies $(11,12,14,15,22-34)$, including 8 RCTs $(14,15,22-27)$, and 9cohort studies $(11,12,28-34)$, and 9919 patients involved in total. Our research showed that lower risk of total haemorrhage events, minor haemorrhage events and thrombosis events in pharmacist-led management group, but the percentage of time within target therapeutic range and the percentage of time within the expanded therapeutic range, major haemorrhage events and mortality were not significantly different between pharmacist-led management model and other management models. Owing to different results were detected of RCTs and observational studies, we adopted GRADE system to rate these evidences from our study. Insufficient sample size and lacking of blinding that led to risk of bias and imprecision resulted in downgrading of most of evidences from RCTs. Whereas, large magnitude of effect upgraded of some evidences from observational studies. According to strict rating of evidences, we declined that results with higher evidence grade were more reliable.

Increased percentage of time in the therapeutic range correlates with improved patient outcomes and lower costs (39). Besides, a marked benefit was found against stroke and total vascular events for oral anticoagulation therapy patients whose TTR above $65 \%$ and the population-average model suggested a minimum target TTR of $60 \%$ to $65 \%$ (40). It's obvious that TTR control was vital for patients with oral anticoagulation therapy. We found that TTR control was significant better in pharmacist-led management group than control groups from overall results $(p=0.007)$ and observational studies pooled results $(p=0.000$, lowquality evidence), while no significant difference between two groups in TTR were detected from RCTs results $(p=0.548)$ as moderate-quality evidence. We concluded that TTR were not significant higher in pharmacist-led management group. On the contrary, Chan et al investigated that a significant difference between pharmacistmanaged care and other management models for the percentage of time within the standard therapeutic range $(p<0.00001)$ (24). One of reasonable explanations of the difference was controversial data conversion. Wilson et al study reported mean TTR with $95 \% \mathrm{CI}$ of two groups and one half of the difference between upper and lower limits of the 95\%CIs were regarded as SD-values in Chan et al study, while our study excluded the unreliable conversion data of Wilson et al which led to reversed results $(24,26)$. Whereas, Witt et al study that included 6645 patients reported significant higher TTR in clinical pharmacy anticoagulation service group compared with usual care $(63.5 \%$ vs $55.2 \%$, $p<0.001)$ which was other from RCTs pooled results in our study (12). The similar results reported in several studies that time in the INR therapeutic range was $48.1 \% \sim 78.5 \%$ in pharmacist management group while $4.01 \% \sim 66.2 \%$ of other management models $(27,28,32,41,42,44,45)$. Most of opposite results came from retrospective chart review studies and our 
results from RCTs grading as moderate quality evidence were more convictive.

As to the percentage of time within the expanded therapeutic range, our pooled results were no significant differences between two groups from 3 RCTs and overall results. A couple of studies indicated that the percentage of time within the expanded therapeutic range of patients in pharmacist-led management group were significantly higher than of control group $(26,41$, 42), which was up to $90.8 \%$ in pharmacist management group from Young et al study $(90.8 \%$ vs $84.8 \%, p<0.0001$ ) (32). Although, no publication bias and serious heterogeneity were detected in RCTs and observational studies of INR control, inadequate studies and sample size arrived at poor results. In that, high quality RCTs with large sample size and long-term follow-up period were expected to provide reliable evidence of INR control by pharmacist -led management.

Haemorrhage is the most common adverse drug reaction associated with warfarin therapy, which is concerned a lot in anticoagulation management. A significant difference in total haemorrhage events between pharmacist-led management group and control group was detected from 3 observational studies with 602 patients as moderate-quality evidence. Compared with low-quality evidence of RCTs results, we concluded that lower risk of total haemorrhage events in patients with pharmacist-led management group. Witt et al reported a significant reduction in all haemorrhage events of pharmacistmanaged anticoagulation clinic group (RR 8.2, 95\%CI: 1.7-39.6, $p=0.009$ ) (12), and Saokaew et al found that pharmacist-participated warfarin-therapy management group had statistically significant effects on prevention of total bleedings (RR: 0.51, 95\% CI: 0.28-0.94, $p=0.019$ ) from RCT analysis (12), which were identical with our study. Whereas, Zhou et al study included data to total haemorrhage events outcome from Bungard et al study that reported the emergency department visits and hospitalizations related to haemorrhage complications, which was not strictly enough to our consideration and we excluded those data $(17,22)$. And difference in data included led to different results with our study.

Patients in pharmacist-led management group had significant lower risk of minor haemorrhage events from the observational studies results of our study as moderate-quality evidence, while pooled results of RCTs were low-quality evidence according to GRADE guidelines. However, Poon et al reported pharmacist-managed oral anticoagulation therapy led to a significant higher risk of minor bleeding events (44). Differences between pharmacist-led management and other management models in documentation and follow-up style might result in bias of information and recall. Otherwise, most of minor haemorrhage events were imperceptible and reported by patients to researchers which might bring about incomplete records. Pharmacists paid more attention on haemorrhage events and patients managed by pharmacists recognized and reported minor haemorrhage events more frequently thanks to systematic patient education. On the basis of our results, less minor haemorrhage events and total haemorrhage events of patients with pharmacist-led management may result in better patient compliance and potentially benefit patients with oral anticoagulation therapy.

As to major haemorrhage events, we found that no significant difference between pharmacist management group and other management models in overall and both RCTs and observational studies results. Due to risk of bias in RCTs and imprecision of RCTs and observational studies, our pooled results provided low to very low evidence according to GRADE guidelines (20). On the contrary, Saokaew et al found that pharmacist-participated warfarin-therapy management was significantly associated with $51 \%$ reduction major bleedings compared with usual care (RR: 0.49, 95\% CI:0.26-.093, $p=0.030)$ with a medium level of heterogeneity $\left(\mathrm{I}^{2}=46.7 \%, p=0.044\right)(16)$. Imparity of the definition of haemorrhage events that derived from included studies could lead to different results, especially the definition of major haemorrhage events. Various bleeding symptoms and signs were described as major, significant, life-threatening and fatal haemorrhage events in different definitions and we pooled and conversed original data according to the definition of major haemorrhage by ISTH in order to restrict major haemorrhage events outcome more precisely and more reliable result (36).

Our study revealed that thrombosis events were significantly reduced in pharmacist-led management group from overall pooled results and observational studies results, while RCTs results detected no significant difference between two groups. And systematic review from Zhou et al which limited in RCTs was identical to our RCTs result. On account of serious risk of bias and imprecision, evidence of thrombosis events from RCTs was low-quality evidence, which was inconsistent with Zhou et al assessment (17). On the other hand, large magnitude of effect upgraded evidence of thrombosis events from observational studies to moderate quality. And we were inclined to believe that pharmacist-led 
anticoagulation management decreased the risk of thrombosis events. Non-randomized controlled trails (quasi-experimental studies and cohort studies) analysis of Saokaew et al study supported that significant reduction of thrombosis events in pharmacist-participated warfarin therapy management group without conspicuous heterogeneity which was in line with our study (16).

The mortality was no significant between pharmacist-led anticoagulation management and other management models in our meta-analysis which was consistent with two former studies (16, 17). Neither the warfarin-related mortality was no significant between pharmacist-participated warfarin therapy management and usual care from RCT (RR:0.65, 95\%CI:0.18-2.42, $p=0.524$ ) and non-RCT (RR:0.77, 95\%CI: 0.39-1.53, $p=0.459$ ) analysis (16). While the follow-up length of our included studies was 90 days to 6 months, long-term studies that provided sufficient evidence on warfarin-related and all-cause death in different anticoagulation management models were expected.

Despite of effectiveness and adverse drug events, costs of different management models were considerable for the long-term anticoagulation therapy. A potential cost avoidance from preventing hospital admissions and emergency room visits for the complications was $\$ 4072.68 /$ person-year of AMS follow-up in Wilt et al study (1995) (45). The cost saving was probably due to the decreased running cost of follow-up and reduction of haemorrhage and thrombosis events in pharmacistled management group. And we expected further studies that evaluated cost-effectiveness of pharmacist-led anticoagulation management model.

To our best knowledge, our review is the newest meta-analysis and systematic review on pharmacist anticoagulation management that included high quality RCTs and observational studies. Compared with former reviews, we searched relevant articles more comprehensively and updated some new and high quality studies (29-34) into the review. We reviewed outcomes TTR, TER, haemorrhage events (total, minor, major), thrombosis events, mortality, patient satisfaction and cost saving, which were more considerately and precisely than former reviews. We also concentrated on strict data extraction, cautious data conversion and coordinating definitions of outcomes between included studies, which would lead to different results of TTR and haemorrhage events from Zhou et al review (17). Moreover, former review (16) came to conclusions from the pooled results with unclear quality of evidence. With the purpose of explaining pooled results scientifically, the acknowledged system on rating quality of evidence GRADE was adopted in our study objectively and rigidly.

We proved that patients with pharmacist-led anticoagulation management had lower risk of total and minor haemorrhage events and thrombosis events with a tendency of lower medical cost. Moreover, limitations of our study were noted following. First, we didn't classify different oral anticoagulation management models in control groups and different pharmacist management models, which could be a complicated analysis that need more high quality studies in the future. Second, differences of races were not considered by subgroup analysis, in that warfarin doses were different between various races from genetic background. Third, there were great disparity in sample size of included studies, most of included studies were performed with small sample size. Four, included RCTs were at high risk of blinding of participants and personnel and unclear risk of blinding of outcome assessment which probably led to risk of bias. Five, heterogeneity between included studies in some outcomes should be interpreted with caution.

\section{CONCLUSIONS}

Our study demonstrated that lower risk of total haemorrhage events, minor haemorrhage events and thrombosis events in pharmacist-led anticoagulation management model. Pharmacist-led anticoagulation management showed benefits for warfarin anticoagulation therapy and was accepted as a proven anticoagulation management model in many countries which could be popularized and promoted worldwide.

\section{REFERENCES}

1. MeWilliam A, Lutter R, Nardinelli C. Health Care Saving from Personalizing Medline Using Genetic Testing: The Case of Warfarin. Washington, DC: American Enterprise Institute-Brookings Joint Center for Regulatory Studies; 2006.

2. Eby C. The pharmacogenetics of vitamin K antagonist anticoagulation drugs. In: Wu AHB, Yeo K-TJ, eds. Pharmacogenomic Testing in Current Clinical Practice. New York, NY: Springer Science+ Business Media; 2011:117-138.

3. Linkins LA, Choi PT, Douketis JD. Clinical impact of bleeding in patients taking oral anticoagulant therapy for venous thromboembolism: a metaanalysis. Ann Intern Med, 2003; 139(11): 893-900. 
4. Kearon C, Akl EA, Comerota AJ, et al. Antithrombotic therapy for VTE disease: antithrombotic therapy and prevention of thrombosis, 9th ed: American College of Chest Physicians evidence-based clinical practice guidelines. Chest, 2012; 141(2 Suppl): e419S-e494S.

5. Lockhart PB, Gibson J, Pond SH, et al. Dental management considerations for the patient with an acquired coagulopathy. Part II. Coagulopathies from drugs. Br Dent J, 2003;195:495-501.

6. Beyth RJ, Quinn LM, Landefeld CS. Prospective evaluation of an index for predicting the risk of major bleeding in out-patients treated with warfarin. Am J Med, 1998; 105(2):91-99.

7. Gitter MJ, Jaeger TM, Petterson TM, et al. Bleeding and thromboembolism during anticoagulant therapy: a population-based study in Rochester, Minnesota. Mayo Clin Proc, 1995; 70(8):725-733.

8. Steffensen FH, Kristensen K, Ejlersen E, et al. Major haemorrhagic complications during oral anticoagulant therapy in a Danish population-based cohort. J Intern Med, 1997;242(6):497-503.

9. Willey VJ, Bullano MF, Hauch O, et al, Management patterns and outcomes of patients with venous thromboembolism in the usual community practice setting. Clin Ther, 2004;26(7):1149-1159.

10. Cortelazzo S,Finazzi G, Viero P, et al. Thrombotic and hemorrhagic complications in patients with mechanical heart valve prosthesis attending an anticoagulation clinic. Thromb Haemost, 1993;69(4):316-320.

11. Chiquette E, Amato MG, Bussey HI. Comparison of an anticoagulation clinic with usual medical care: anticoagulation control, patient outcomes, and health care costs. Arch Intern Med,1998;158(15):16411647.

12. Witt DM, Sadler MA, Shanahan RL, et al. Effect of a centralized clinical pharmacy anticoagulation service on the outcomes of anticoagulation therapy. Chest, 2005;127(5):1515-1522.

13. Ansell J, Hirsh J, Dalen J, et al. Managing oral anticoagulant therapy. Chest, 2001;119:22S-38S.

14. Lalonde L, Martineau J, Blais N, et al. Is long-term pharmacist-managed anticoagulation service efficient? A pragmatic randomized controlled trial. Am Heart J, 2008; 156(1):148-154.

15. Verret L, Couturier J, Rozon A, et al. Impact of a pharmacist-led warfarin self-management program on quality of life and anticoagulation control: a randomized trial. Pharmacotherapy, 2012;32(10):871-879.

16. Saokaew S, Permsuwan U, Chaiyakunapruk N, et al. Effectiveness of pharmacist-participated warfarin therapy management: a systematic review and metaanalysis. J Thromb Haemost, 2010;8(11):2418-2427.

17. Zhou S, Sheng XY, Xiang Q, et al. Comparing the effectiveness of pharmacist-managed warfarin anticoagulation with other models: a systematic review and meta-analysis. J Clin Pharm Ther, 2016;
41(6):602-611.

18. Higgins JGS. Cochrane handbook for systematic reviews of interventions version 5.1.0 [updated March 2011]. The Cochrane Collaboration [EB/OL]. Available at http://www.cochrane-handbook.org.

19. Wells GA, Shea B, O'Connell D, et al. The Newcastle-Ottawa Scale (NOS) for assessing the quality of nonrandomized studies in meta-analysis. Ottawa Hospital Research Institute website. Available http://www.ohri.ca/programs/clinical_epidemiology/ oxford.asp.

20. Guyatt GH, Oxman AD, Vist GE, et al. GRADE: an emerging consensus on rating quality of evidence and strength of recommendations. BMJ, 2008;336(7650):924-926.

21. Higgins JGS, Thompson SG, Deeks JJ, et al. Measuring inconsistency in meta-analyses. BMJ, 2003;327(7414):557-560.

22. Bungard TJ, Ritchie B, Garg S, et al. Sustained impact of anticoagulant control achieved in an anticoagulation management service after transfer of management to the primary care physician. Pharmacotherapy, 2012;32(2):112-119.

23. Jackson SL, Peterson GM, Vial JH, et al. Improving the outcomes of anticoagulation: an evaluation of home follow-up of warfarin initiation. J Inter Med, 2004;256(2):137-144.

24. Chan FW, Wong RS, Lau WH, et al. Management of Chinese patients on warfarin therapy in two models of anticoagulation service- a prospective randomized trial. Br J Clin Pharmacol, 2006;62(5):601-609.

25. Schillig J, Kaatz S, Hudson M, et al. Clinical and safety impact of an inpatient pharmacist-directed anticoagulation service. J Hosp Med, 2011;6(6):322328.

26. Wilson SJ, Wells PS, Kovacs MJ, et al. Comparing the quality of oral anticoagulant management by anticoagulation clinics and by family physicians: a randomized controlled trial. CMAJ, 2003;169(4):293-298.

27. Lakshmi R, James E, Kirthivasan R. Study on impact of clinical pharmacist's interventions in the optimal use of oral anticoagulants in stroke patients. Indian J Pharm Sci, 2013;75(1):53-59.

28. Airee A, Guirguis AB, Mohammad RA. Clinical outcomes and pharmacists' acceptance of a community hospital's anticoagulation management service utilizing decentralized clinical staff pharmacists. Ann Pharmacother, 2009;43(4):621-628.

29. Elewa H, Jalali F, Khudair N, et al. Evaluation of pharmacist-based compared to doctor-based anticoagulation management in Qatar. J Eval Clin Pract, 2016;22(3):433-438.

30. Sargent R, Brocklebank C, Tam-Tham $H$, et al. Advantages of a warfarin protocol for long-term care pharmacists: a retrospective cohort study. Can Geriatr J, 2016;19(2):40-49.

31. Thanimalai S, Shafie AA, Hassali MA, et al. 
Comparing effectiveness of two anticoagulation management models in a Malaysian tertiary hospital. Int J Clin Pharm, 2013(5);35:736-743.

32. Young S, Bishop L, Twells L, et al. Comparison of pharmacist managed anticoagulation with usual medical care in a family medicine clinic. BMC Fam Pract, 2011;12:88.

33. Hall D, Buchanan J, Helms B, et al. Health care expenditures and therapeutic outcomes of a pharmacist-managed anticoagulation service versus usual medical care. Pharmacotherapy, 2011;31(7):686-694.

34. Martin CY, Hermann SA, Couldry RJ, et al. Impact of pharmacist-managed warfarin on patient outcomes in an academic medical center. Am J Health Syst Pharm,2015;72:(Supp11): $\quad$ S31. doi: 10.2146/sp150008.

35. Rosendaal FR, Cannegieter SC, van der Meer FJ, et al. A method to determine the optimal intensity of oralanticoagulant therapy. Thromb Haemost, 1993; 69(3):236-239.

36. Schulman S, Kearon C. Definition of major bleeding in clinical investigations of antihemostatic medicinal products in non-surgical patients. J Thromb Haemost, 2005;3(4):692-694.

37. Sawicki PT. A structured teaching and selfmanagement program for patients receiving oral anticoagulation: a randomized controlled trial. JAMA, 1999;281(2):145-150.

38. Samsa G, Matchar DB, Dolor RJ, et al. A new instrument for measuring anticoagulation-related quality of life: development and preliminary validation. Health Qual LifeOutcomes, 2004;2:22.

39. Baker WL, Cios DA, Sander SD, et al. Meta-analysis to assess the quality of warfarin control in atrial fibrillation patients in the United States. J Manag Care Pharm, 2009; 15:244-252.

40. Connolly SJ, Pogue J, Eikelboom J, et al. Benefit of oral anticoagulant over antiplatelet therapy in atrial fibrillation depends on the quality of international normalized ratio control achieved by centers and countries as measured by time in therapeutic range. Circulation, 2008;118(20):2029-2037.

41. Saokaew S, Sapoo U, Nathisuwan S, et al. Anticoagulation control of pharmacist-managed collaborative care versus usual care in Thailand. Int J Clin Pharm, 2012;34(1):105-112.

42. Gupta V, Kogut SJ, Thompson S. Evaluation of differences in percentage of international normalized ratios in range between pharmacist-led and physician-led anticoagulation management services. J Pharm Pract, 2015;28(2):249-255.

43. Harrison J, Shaw JP, Harrison JE. Anticoagulation management by community pharmacists in New Zealand: an evaluation of a collaborative model in primary care. Int J Pharm Pract, 2015;23(3):173-181.

44. Poon IO, Lal L, Brown EN, et al. The impact of pharmacist-managed oral anticoagulation therapy in older veterans. J Clin Pharm Ther, 2007;32(1):21-29.
45. Wilt VM, Gums JG, Ahmed OI, et al. Outcome analysis of a pharmacist-managed anticoagulation service. Pharmacotherapy, 1995;15(6):732-739. 
J Pharm Pharm Sci (www.cspsCanada.org) 20, 378 - 396, 2017 